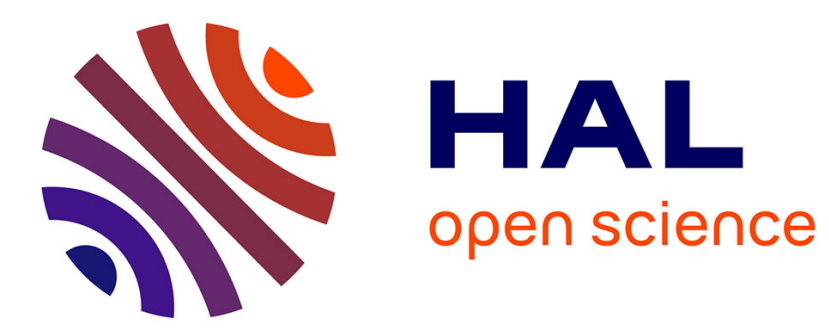

\title{
On the optical constants of metals at wavelengths shorter that their critical wavelengths
}

W.R. Hunter

\section{To cite this version:}

W.R. Hunter. On the optical constants of metals at wavelengths shorter that their critical wavelengths. Journal de Physique, 1964, 25 (1-2), pp.154-160. 10.1051/jphys:01964002501-2015400 . jpa00205728

\section{HAL Id: jpa-00205728 https://hal.science/jpa-00205728}

Submitted on 1 Jan 1964

HAL is a multi-disciplinary open access archive for the deposit and dissemination of scientific research documents, whether they are published or not. The documents may come from teaching and research institutions in France or abroad, or from public or private research centers.
L'archive ouverte pluridisciplinaire HAL, est destinée au dépôt et à la diffusion de documents scientifiques de niveau recherche, publiés ou non, émanant des établissements d'enseignement et de recherche français ou étrangers, des laboratoires publics ou privés. 


\title{
ON THE OPTICAL CONSTANTS OF METALS AT WAVELENGTHS SHORTER THAT THEIR CRITICAL WAVELENGTHS (1)
}

\author{
By W. R. HUNTER,
}

E. O. Hulburt Center for Space Research, U. S. Naval Research Laboratory, Washington, D. C.

\begin{abstract}
Résumé. - On a étudié les propriétés optiques de $\mathrm{Al}, \mathrm{In}, \mathrm{Mg}$ et $\mathrm{Si}$ dans l'extrême ultra-violet pour des longueurs d'onde inférieures à la longueur d'onde critique. On a trouvé que $\mathrm{Al}, \mathrm{Mg}$ et $\mathrm{Si}$, qui ont des électrons de valence faiblement liés et des électrons internes fortement liés, peuvent être décrits, avec une bonne approximation, par la théorie des électrons libres. Pour l'indium, dans ce domaine spectral, le terme dominant de la constante diélectrique complexe est le terme dû aux électrons libres; cependant, d'autres mécanismes d'absorption ne sont pas négligeables, aussi on ne peut pas utiliser une simple théorie à deux paramètres.
\end{abstract}

Abstract. - The optical properties of $\mathrm{Al}, \mathrm{In}, \mathrm{Mg}$, and $\mathrm{Si}$ have been investigated in the extreme ultraviolet at wavelengths shorter than their critical wavelengths. It was found that $\mathrm{Al}, \mathrm{Mg}$, and $\mathrm{Si}$, which have loosely bound valence electrons and tightly bound core electrons can be described, to a good approximation, by the free electron theory. For In, in this spectral range, the dominant term in the complex dielectric constant is the free electron term, however, other absorption mechanisms are not negligible so a simple two-parameter thoery cannot be used.

Introduction. - One of the consequences of Maxwell's theory of the propagation of electromagnetic waves in a medium containing free charges is the existence of a critical wavelength $\lambda_{c}$. Wavelengths greater than $\lambda_{c}$ are reflected while the shorter wavelengths penetrate into the medium. A simple explanation of $\lambda_{c}$ is that the free charges oscillate in phase with the electromagnetic waves for $\lambda>\lambda_{c}$ but are $\pi$ radians out of phase for $\lambda<\lambda_{c}$. Thus the energy reradiated by the oscillating charges interferes constructively with the transmitted wave and destructively with the reflected wave for $\lambda<\lambda_{0}$ and vice versa.

In the case of a metal, the free charges are electrons which, by virtue of their coulomb interactions, are capable of collective oscillations. Consequently it is to be expected that metals will have a $\lambda_{c}$ given by ;

$$
\lambda_{\mathrm{c}}=\frac{c}{\mathrm{e}} \sqrt{\pi / N}
$$

where $c$ is the velocity of light, $e$ is the electronic charge and $N$ the valence electron density.

This simple model was used by Zener [1] to explain the experimental results of Wood [2] who showed that the alkali metals are transparent in the ultraviolet. This theory was successful in that it gave fair approximations to the observed wavelength at which transmission set in. However, it requires total reflection at all angles of incidence for $\lambda>\lambda_{c}$ which is not the case. Kronig [3] modified Zener's theory by introducing damping of the free electron motion, due to collisions with the

(1) Supported, in part, by the National Aeronautics and Space Administration. lattice. This then becomes the free electron theory of Drude [4] and, for the purpose of describing optical phenomena, can be put into the following form :

$$
\begin{aligned}
& \varepsilon_{1}=n^{2}-k^{2}=1-\frac{\omega_{c}^{2} \tau^{2}}{1+\omega_{c}^{2} \tau^{2}} \\
& \varepsilon_{2}=2 n k=\frac{1}{\omega_{c} \tau} \frac{\omega_{c}^{2} \tau^{2}}{1+\omega^{2} \tau^{2}} .
\end{aligned}
$$

In the limit as $\tau$ approaches infinity, eq. (1) becomes :

$$
\varepsilon_{1}=n^{2}=1-\left(\omega_{\mathrm{c}}^{2} / \omega^{2}\right)
$$

and eq. (2) becomes zero, i.e., Zener's model. $\varepsilon_{1}$ and $\varepsilon_{2}$ are the real and imaginary parts of the complex dielectric constant, respectively ; $n$ is the index of refraction, $k$ is the extinction coefficient, $\omega_{c}$ is the critical angular frequency and $\tau$ is the relaxation time of the electrons.

A Drude type model describes fairly well the optical properties of the alkali metals which have a loosely bond valence electron and tightly bound core electrons [5]. Hence, it is reasonable to expect that the theory would be at least a good first approximation to other metals that have loosely bound valence electrons and tightly bound cores. Pines [6] discussed the classification of metals by the binding energy of the valence and core electrons and found that the metals listed in Table I have this characteristic in common with the alkali metals. Thus the inference can be made that a critical wavelength exists for these metals at which transmission should commence. In Table $I$ is listed, for several elements, the value for $\lambda_{0}$ and the nearest absorption edge at which $k$ should once 
TABLE I

Critical angle and closest absorption edge for metals with loosely bound valence AND TIGHTLY BOUND CORE ELEGTRONS

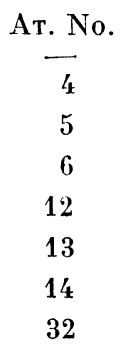

\begin{tabular}{lr}
\multicolumn{1}{c}{ ELEm EN } & \multicolumn{1}{c}{$\lambda_{\mathrm{c}}(\AA)$} \\
Beryllium & $639\left({ }^{1}\right)$ \\
Boron & $650\left({ }^{2}\right)$ \\
Carbon & $560\left({ }^{2}\right)$ \\
Magnesium & $1198\left({ }^{1}\right)$ \\
Aluminum & $837\left({ }^{1}\right)$ \\
Silicon & $762\left({ }^{1}\right)$ \\
Germanium & $743\left({ }^{1}\right)$
\end{tabular}

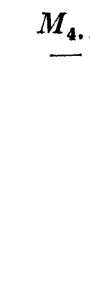

\begin{tabular}{ll}
$M_{1}$ & $L_{2.3}$ \\
\hline
\end{tabular}

$110 . \bar{K}$

$66.3 \AA\left({ }^{3}\right)$

$43.6 \AA(3)$

$247.9 \AA\left(^{3}\right)$

$170.0 \AA(3)$

$126.5 \AA(3)$

$470 \AA\left(^{4}\right) \quad 70 \AA\left({ }^{3}\right)$

(1) [14] R. LaVilla, Private communication.

(2) [6] D. Pines.

(3) [28] Handbook of Chemistry and Physics.

(4) [29] A. E. Sandström.

again become large enough to reduce or prevent transmission.

Metals that cannot be described by. the free electron theory may still have a $\lambda_{c}$ accompanied by transmission for $\lambda<\lambda_{c}$. This means that the free electron term in the complex dielectric constant is dominant in the region around $\lambda_{c}$, however, other absorption processes will not be far enough removed from the region to be negligible. Some examples are indium, tin, antimony, and bismuth.

Other metals, such as gold [7], have no optical transmission and, therefore, no trace of a $\lambda_{c}$, although it can be predicted on the basis of theory.

If the metal becomes transparent for $\lambda<\lambda_{c}$, $k$ must be rather small. Furthermore, from a consideration of eq. (3), $n$ will be real but less than unity, so that radiation can center the medium only at angles less than the critical angle, $\alpha_{c}$. Hence, for $\lambda<\lambda_{c}$ metals that have transmission bands or regions will be expected to have a very small $k$ and $n$ less than unity.

Experimental methods. - The metals to be investigated were in the form of thin evaporated films, deposited under optimum conditions as described by Hass, Hunter, and Tousey [8]. In the case of unbacked films, a suitable parting agent was placed on the substrate prior to the evaporation of the metal, and another substrate, situated alongside, was exposed during the evaporation and was subsequently coated with silver and used to measure the film thickness by multiple beam interferometry. Details of the process of making unbacked films will be published [9].

The extinction coefficient, $k$, was measured by transmission through unbacked metal films. By measuring the transmittance of films of different thicknesses, absorption at the surface by an oxide layer, and also reflection losses, can be eliminated, assuming they are the same in both cases. The formula for obtaining $k$ by this method is :

$$
k=\frac{\lambda}{4 \pi\left(x_{2}-x_{1}\right)} \ln _{\mathrm{e}}\left(T_{1} / T_{2}\right)
$$

where $x$ is the film thickness and $T$ the transmittance.

If $k$ is very small, and $n$ is less than unity, it is possible to use a technique similar to the critical angle method for measuring the index of nonabsorbing solids and liquids in the visible region. Calculations of reflectance versus angle of incidence for a medium with $n=0.707, \alpha_{\mathrm{c}} \cong 45^{\circ}$ and

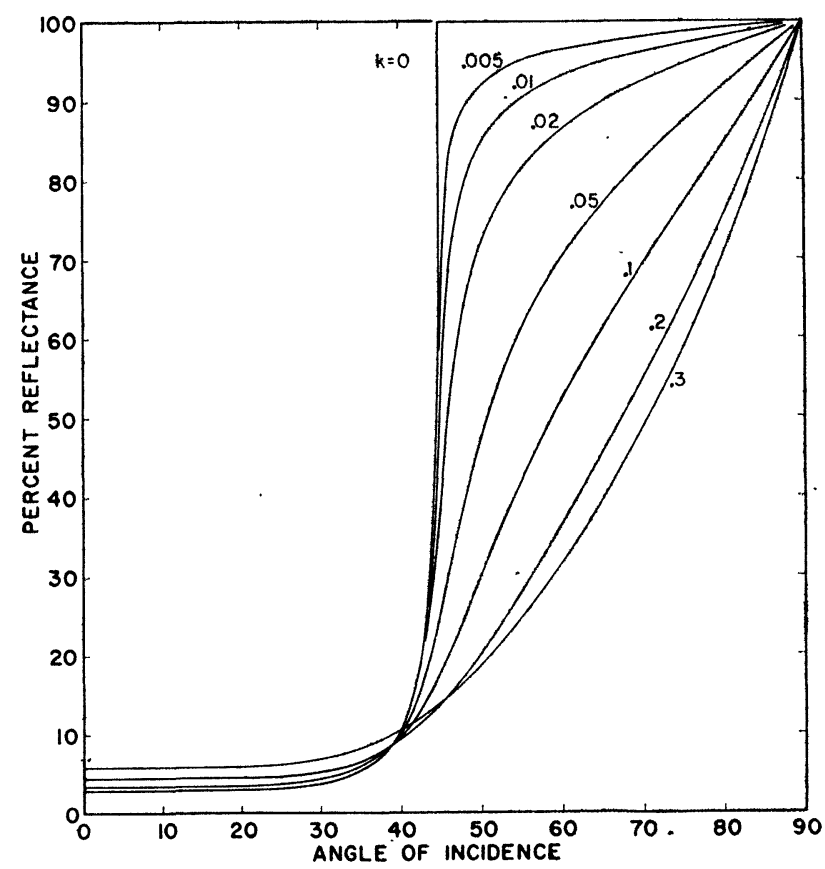

Fig. 1. - Calculated reflectance versus angle of incidence for $n=0.707, \alpha_{c} \cong 45^{\circ}$, and different values of $k$. 
different values of $k$, shown in figure 1 , illustrate that a definite $\alpha_{c}$ can be observed only if $k=0$, however, for small values of $k$ there is a sudden drop in reflectance in the region of $\alpha_{c}$. The index, $n$, was obtained from the position of maximum slope of the reflectance versus angle of incidence curve. The position of the angle of maximum slope, $\alpha_{\mathrm{m}}$, of the reflectance curves is very close to $\alpha_{c}$, but moves further away as $k$ increases. Eventually $k$ becomes large enough so that the $R$ vs $\alpha$ curve is monotonic, as is the case of $k>0.2$.

Further calculations were made to find the dependence of $\alpha_{m}$ on $k$. The results are shown in figure 2, where the angle of incidence of $\alpha_{m}$ is shown as a function of $k$ for different values of the

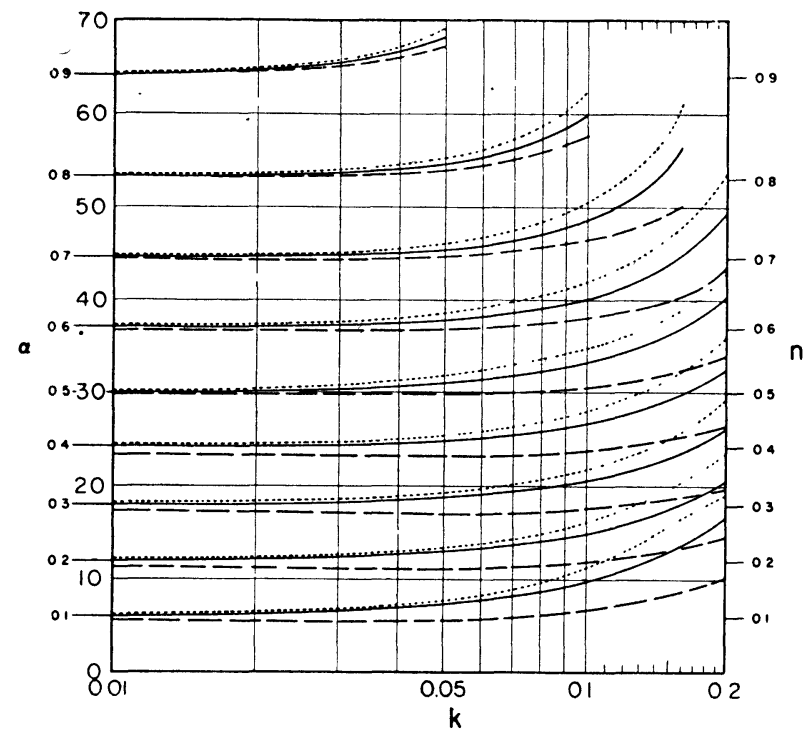

Fig. 2. - Calculated position of the angle of maximum slope, $\alpha_{\mathrm{m}}$, as a function of $k$. The full lines are for unpolarized radiation, the dotted lines for the $\mathrm{p}$-component and the dashed lines for the $s$-component.

index. The curves for $n \geqq 0.7$ are terminated when the respective $R$ vs $\alpha$ curves become monotonic. By means of these curves a correction for the value of $n=\sin \alpha_{\mathrm{m}}$ can be determined if $k$ is known for the material. For example, if the measured value of $\alpha_{\mathrm{m}}=49.5^{\circ}$, and $k=0.2$ (fig. 2 ) indicates that $n=0.6$; neglect of the correction would yield $n=0.76$, an error of about $27 \%$. Polarization would cause the measured $n$ to vary between 0.68 for complete $s$-polarization and 0.81 for complete $\mathrm{p}$-polarization, corresponding to errors of $15 \%$ and $33 \%$, respectively. This is, of course an extreme case ; corrections for $k \neq 0$ are usually quite small.

The effect of reflections from the melal-substrate interface and the oxide layer are discussed elsewhere [10] and were shown to be, for the most part, negligible.
Results. - The results for aluminum are shown in figure 3. In the upper part of the figure, the dots through which a solid line has been drawn, represent measured values of $\sin \alpha_{\mathrm{m}}$. The large shaded circles, at $584 \AA$ and $736 \AA$, are the results of previous measurements [11] using an interfer-

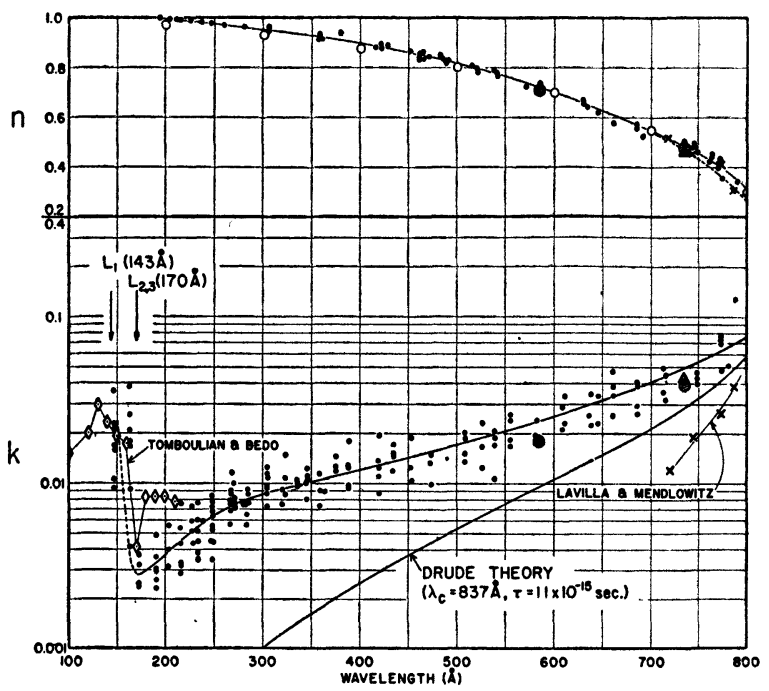

FIG. 3. - Optical constants of aluminum at wavelengths shorter than the critical wavelength.

ence technique. At the same wavelengths, the triangles are data obtained by Madden, Canfield, and Hass [12] from oxide-free surfaces using the general reflectance method. Data obtained by LaVilla and Mendlowitz [13] from inelastic electron scattering experiments are shown as crosses.

Index measurements could only be carried out to $200 \AA$ because, at shorter wavelengths, $\alpha_{\mathrm{m}}>84^{\circ}$, which was the largest angle at which measurements could be made. The open circles represent $n$ calculated from the free electron theory using $\lambda_{c}=837 \AA$ and $\tau=1.1 \times 10^{-15}$ seconds [14], and are in very good agreement with the measured values. From $700 \AA$ to longer wavelengths, the broken curve represents the correction necessary because of the displacement of $\alpha_{m}$ by the everincreasing $k$.

In the lower half of the figure are shown the measured values of $k$. The scatter of points is quite large, especially at wavelengths shorter than the $L_{2,3}$ x-ray edge at $170 \AA$, where the dotted line is to be regarded merely as an indication of the behavior of $k$. For $\lambda>170 \AA$, the results are somewhat more consistent and the scatter of data decreases with increasing wavelength. Once again the large shaded circles represent the results of previous measurements by transmission while the crosses and triangles are data of LaVilla and Mendlowitz and Madden, Canfield, and Hass, respectively. The diamonds represent the data of 
Tomboulian and Bedo [15], obtained photographically using a synchroton as a light source. Values of $k$, calculated using the free electron theory, are so labelled. There is a definite departure from the free electron model in that the measured $k$ does not decrease with decreasing wavelength as rapidly as the calculated values. This is due to the fact that interband transitions at approximately $8000 \AA$ which are not included in the Drude theory are still exerting a slight influence.

The data of Tomboulian and Bedo show a sharp dip in the value of $k$ at the $L_{\mathbf{2} \cdot 3}$ edge. Astoin and Vodar [16], who have measured the transmittance of aluminum films have reported a sharp peak in transmittance at the $L_{\mathbf{2} .3}$ edge, corresponding to the dip in $k$ observed by Tomboulian and Bedo. Both sets of investigators used aluminum films backed by thin films of zapon or collodion. In the earlier work of Tomboulian and Pell [17] on unbacked aluminum films, $5000 \AA$ thick, the sharp peak was absent. No sharp peak was observed in the present work, although it would have been detected in spite of the large scatter of the points near the edge.

In figure 4 are shown the optical properties of aluminum from $100 \AA$ to $6000 \AA$. Open circles represent values taken from the experimental

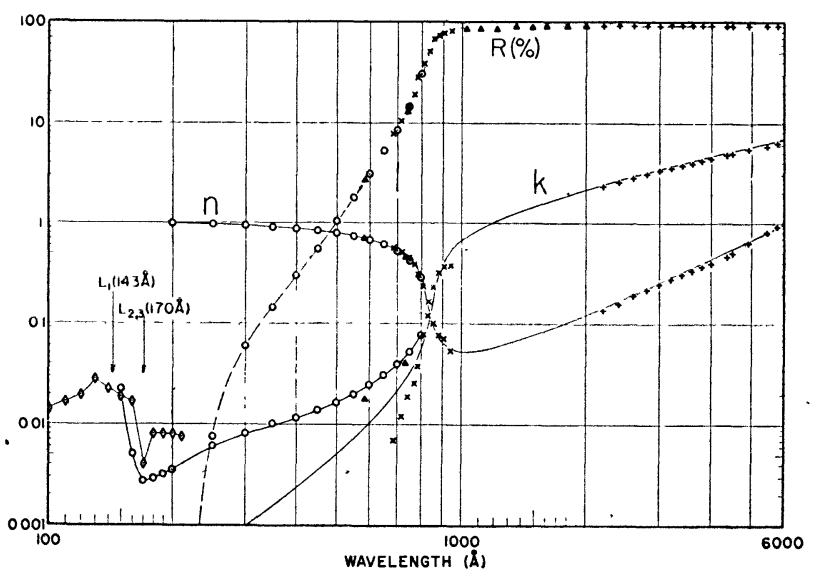

Fig. 4. - Optical constants and normal incidence reflectance of aluminum.

curves of the preceeding figure, otherwise the data symbols have the same meaning as in the previous figure with the exception of the additional data of Hass and Waylonis [18] for the near ultraviolet and visible, shown as plus signs.

The solid line, drawn through the index data, represents the index calculated using the free electron theory with the parameters given above, and is in very good agreement with the experimental data at all wavelengths. Good agreement is also obtained in the visible region between the data points for $k$ and the calculated values, which are shown by the solid line. At wavelengths shorter than about $860 \AA$, however, the curve given by the free electron theory drops more rapidly than the experimental values, shown by the open circles, for the reason given earlier. The dotted line is the reflectance calculated using the free electron theory wich agrees very well with the measured values from $6000 \AA$ to $1025 \AA$. At shorter wavelengths, the reflectance data points, connected by the dashed line, represent calculations from the measured $n$ and $k$ since interference effects present in thin films prevent direct measurements of reflectance.

The true values of $n$ and $k$ still remain to be found in the interval between $1000 \AA$ and $2000 \AA$. Because of the agreement between calculated and measured reflectances, however, it is expected that the measurements will not show large deviations from those represented by the solid lines.

Indium has two distinct spectral regions where the value of $k$ becomes very small, from $120 \AA$ to shorter wavelengths, the cut-off wavelength could not be determined; and from $744 \AA$ to approximately $1100 \AA$. Apparatus was not available for index measurements at the very short wavelengths so that only measurements of the extinction coefficient were made. The results are shown in Table II. Because of experimental difficulties, the numbers are accurate only to within $50 \%$.

TABLE II

ExtinCtion COEFFICIENT, $k$, AND MASS ABSORPTION COEFFICIENT, $\mu / \rho$, OF INDIUM

$\begin{array}{lcc}\lambda(\AA) & \frac{k}{\mu / \rho} \\ \overline{44.3} & 0.003 & \frac{10^{4}}{67} \\ 67 & 0.003 & 10^{4}\end{array}$

The optical constants of indium from $744 \AA$ to $1085 \AA$ are shown in figure 5 , where measured values are represented by dots. The broken line shows the index curve after correction for the effect of non-zero $k$.

Although the free electron term in the complex dielectric constant is dominant in this region, other terms are not negligible. For example, the sharp increase in $k$, which begins approximately at $760 \AA$, indicates the commencement of an optical absorption process, thus introducing another term into the complex dielectric constant. This corresponds to the sudden termination of transmission observed by Walker, Rustgi, and Weissler [19] and attributed, by them to interband transitions. Hence, no simple two-parameter model can be made to fit the data.

At wavelengths less than $800 \AA$, the location of $\alpha_{m}$ was uncertain and the values shown may be in error by $50 \%$ or more. An attempt was made to obtain more accurate values by matching the cal- 


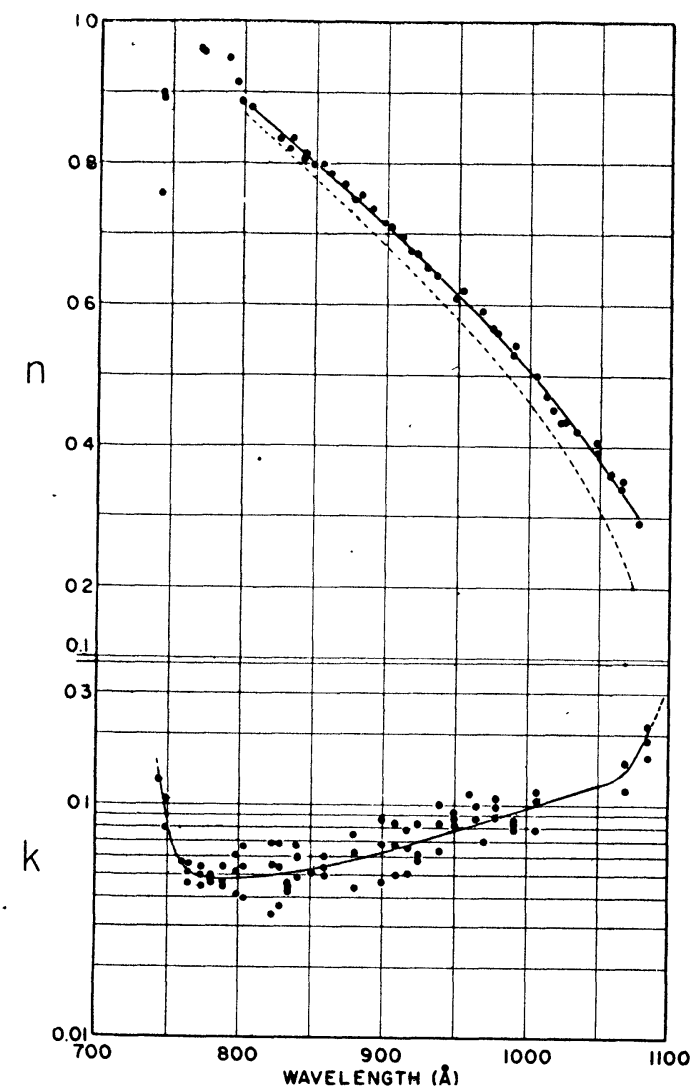

Fig. 5. - Optical constants of indium from $744 \AA$ to $1085 \AA$.

culated transmittance to that which was observed by varying $n$, however, the calculated transmittance was insensitive to the value of $n$ chosen and the attempt was abandoned.

The critical wavelength associated with the free

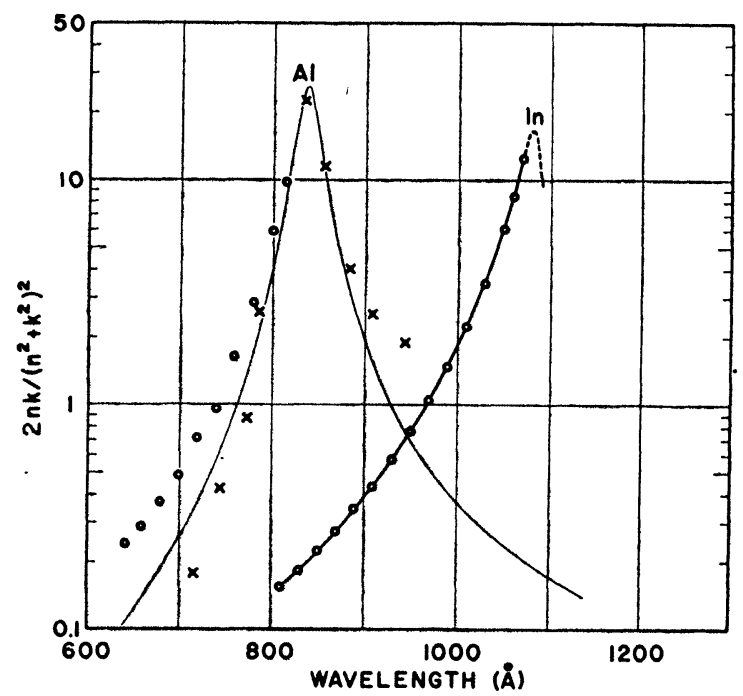

Fig. 6. $-2 n k /\left(n^{2}+k^{2}\right)^{2}$ as a function of wavelength for aluminum and indium. electron term, may be determined from the quantity, $2 n k /\left(n^{2}+k^{2}\right)^{2}[20,21]$ which should have a sharp peak very close to the position of $\lambda_{c}$. In figure 6 , this expression is shown as a function of wavelength for indium and aluminum. The circles represent experimental points obtained in the present experiment, while the crosses, for aluminum, are the data of LaVilla and Mendlowitz. The solid line for aluminum was calculated using $n$ and $k$ obtained from the free electron theory, while the line for indium is only to connect the data points, since no model was available for comparison.

Since, in this experiment, the optical constants of indium were measured at wavelengths shorter than the critical wavelength, the peak of the curve could not be located experimentally. By extrapolating the corrected index curve for indium, however, a peak was found at $1080 \AA$, shown by the dashed line, which agrees very well with the characteristic energy loss reported by Robins [22] at $1097 \AA$. Similarly, for aluminum the optical data extend only to $800 \AA$. However, the data of LaVilla and Mendlowitz agree very closely with the calculated curve which has a peak at approximately $840 \AA$.

The occurrence of optical transmission suggests that $\lambda_{c}$ may be found from the onset in transmission. This a rather inaccurate way in which to determine $\lambda_{c}$ since the thickness of the film and the sensitivity of the measuring equipment essentially determine the longest wavelength at which transmittance occurs. For example, Walker, Rustgi, and Weissler have published transmission curves for aluminum and indium, among other metals, and found the onset of transmission to occur at approximately $855 \AA$ and $1100 \AA$, respectively. In this experiment, transmission measurements could be made to wavelengths as long as 834 and $1085 \AA$ on aluminum and indium, respectively, while Wilkinson [23] has photographed lines close to $1200 \AA$ through indium films.

- Mendlowitz [21] has pointed out that the onset of transmission may not occur at $\lambda_{0}$ but at a somewhat shorter wavelength at which $k$ becomes small enough to permit transmission. He has also investigated the location of the maximum in the quantity, $2 n k /\left(n^{2}+k^{2}\right)^{2}$ and has shown that, for nonzero damping, the peak occurs at a wavelength longer than $\lambda_{0}$. The shift is very small for aluminum, amounting to approximately one part in 5000 , and since the curve for indium is quite similar, it is expected that the maximum is very close to $\lambda_{c}$.

The results for magnesium are shown in figure 7. In the upper part of the figure, the dots through which the solid line has been drawn represent the measurements of $\sin \alpha_{\mathrm{m}}$. The open circles are calculations using the free electron theory with the 


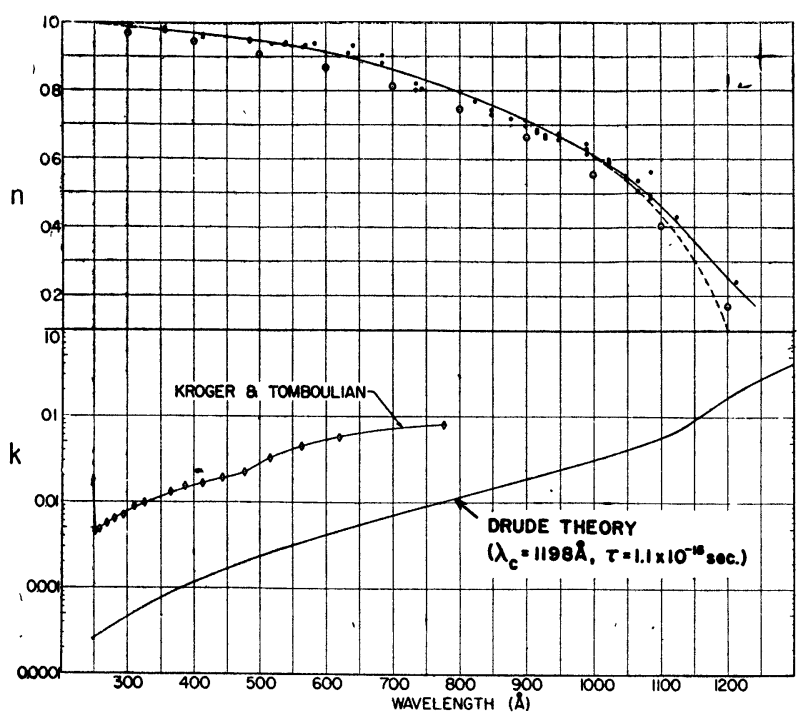

Fig. 7. - Optical constants of magnesium at wavelengths shorter than the critical wavelength.

parameters $\lambda_{c}=1198 \AA$ and $\tau=1.1 \times 10^{-15}[14]$ The calculated values agree fairly well with the measured values, although not as well as in the case of aluminum. The greater departure of the points from the smooth curve from 600 to $800 \AA$ is attributed to uncertainties in the measurements. However, some electron scattering experiments (24) show a broad peak centered at about $20 \mathrm{eV}$, believed to be due to $\mathrm{MgO}$, which may have influenced the results.

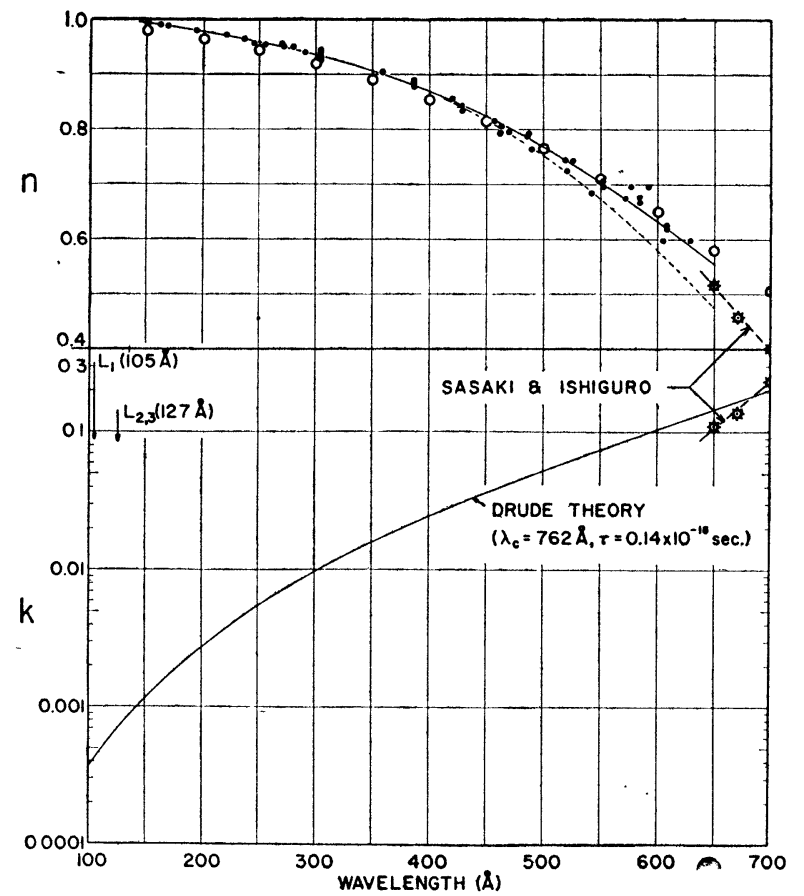

Fig. 8. - Optical constants of silicon at wavelengths shorter than the critical wavelength.
The lower part of the figure shows $k$, calculated using the free electron theory. Kroger and Tomboulian [25] have reported values of $k$, measured using a photographic technique, from $230 \AA$ to $775 \AA$. Their values, which were converted from absorption coefficient $\mu$, to extinction coefficient $k$, are shown as diamonds. The values they reported are approximately one order of magnitude larger than those calculated using the free electron theory. In view of the departure of the measured $k$ values of aluminum from those calculated using the free electron theory this behavior does not seem unreasonable. There is also some evidence of structure in their curve, aside from the $L_{\mathbf{2} .3} \mathrm{x}$-ray edge at approximately $250 \AA$, that could not be expected to appear in calculations using the free electron theory.

Since measured values of $k$ with which to correct the index curve are not available, the calculated values have been used. The corrected curve is shown by the broken line in the upper half on the figure.

The results for silicon are shown in figure 8, with dots and open circles, measured and calculated,

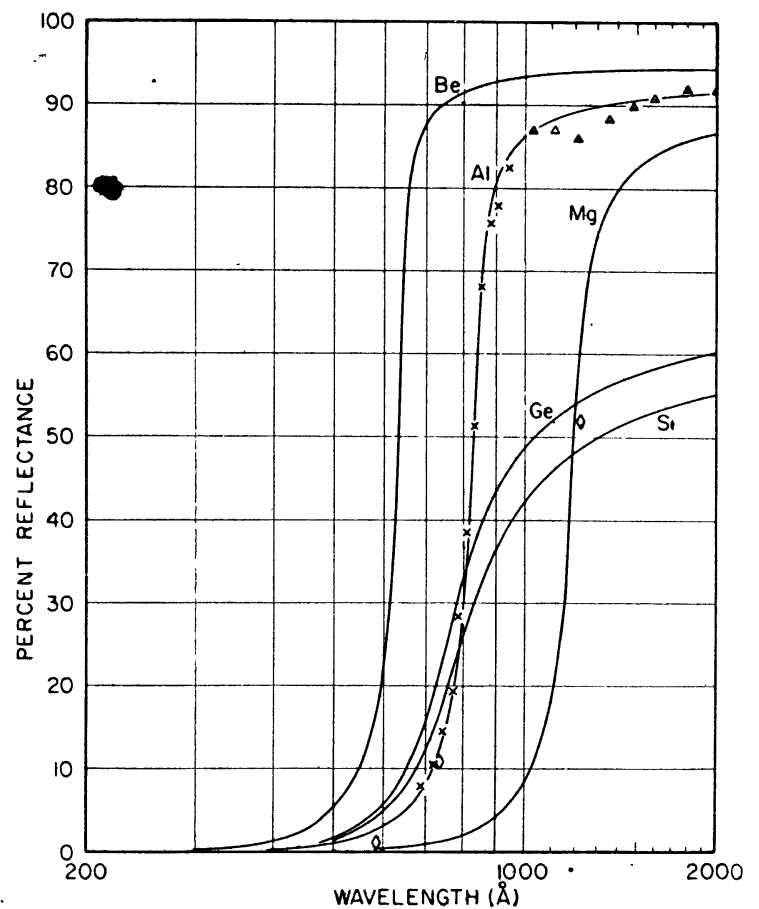

FIG. 9. - Normal incidence reflectance of various metals in the extreme ultraviolet. Data points $\Delta[12]$ and $x$ [13] are for aluminum, and $\Delta[27]$ is for germanium. The solid lines were calculated using a Drude type model with the following parameters [14]:

\begin{tabular}{|c|c|c|c|c|c|}
\hline & $\mathrm{Be}$ & $\mathrm{Al}$ & $\mathrm{Mg}$ & $\mathrm{Ge}$ & $\mathrm{Si}$ \\
\hline $\begin{array}{l}\lambda_{c}(\AA) \\
\tau\left(10^{-15} \mathrm{~s}\right)\end{array}$ & $\begin{array}{l}639 \\
1.3\end{array}$ & $\begin{array}{l}837 \\
1.1\end{array}$ & $\begin{array}{c}1198 \\
1.1\end{array}$ & $\begin{array}{l}743 \\
0.16\end{array}$ & $\begin{array}{r}762 \\
0.14\end{array}$ \\
\hline
\end{tabular}


respectively. The crenulated circles are data of Sasaki and Ishiguro [26]. At the shorter wavelengths the measured and calculated data agree rather well ; however, from approximately $500 \AA$ to longer wavelengths, the corrected index curve drops more rapidly than the calculated values. The corrected curve has the same slope as the data of Sasaki and Ishiguro although the magnitudes are not quite the same.

In the lower part of the figure are shown the calculated values of $k$ using the free electron theory with the parameters indicated. Since the free electron theory does not take $x$-ray edges into account, the curve does not show the sudden increase in $k$ that must exist at the $L_{\mathbf{2}, 3}$ edge and possibly at the $L_{1}$ edge also. The data of Sasaki and Ishiguro fit the calculated values approximately as far as magnitudes are concerned but the slope is different.

Reflectance data for some of the metals listed in
Table I are collected in figure 9 . The solid lines are the reflectance at normal incidence calculated on the basis on the free electron theory using the parameters given in the figure caption. The data points indicate that the approximation is close for aluminum and germanium. For aluminum, the triangles represent the data of Madden, Canfield, and Hass, while the crosses are the calculated reflectances from the data of LaVilla and Mendlowitz. The diamonds represent data tor germanium obtained by Madden [27].

Acknowledgments. - The author is pleased to acknowledge the efforts of D. W. Angel who made the unbacked films of aluminum and indium, S. G. Tilford who discovered the very short wavelength window in indium, and R. L.. Blake, J. F. Meekins, and A. E. Unzicker who measured the transmittance of the indium films at the very short wavelengths.

\section{REFERENCES}

[1] Zener (C.), Nature, 1933, $132,968$.

[2] Wood (R. W.), Nature, 1933, 131, 582 ; Phys. Rev., 1933, 44, 353.

[3] Kronig (R. de L.), Nature, 1934, 133, 211.

[4] Drude (P.), Theory of Optics, Dover Publ. Co., New York, 1959.

[5] Seitz (F.), Modern Theory of Solids, McGraw-Hill Publ. Co., New York, 1940.

[6] Pines (D.), Rev. Mod. Physics, 1956, 28, 194.

[7] Canfield (L. R.), Hass (G.) and Hunter (W. R.), Proceedings of this Colloquium.

[8] Hass (G.), Hunter (W. R.) and Tousey (R.), J. Opt. Soc. Am., 1956, 46, 1009.

[9] ANGel (D. W.), To be published.

[10] Hunter (W. R.), J. Opt. Soc. Am. 1964, 54, 15.

[11] Hass (G.), Hunter (W. R.) and Tousey (R.), J. Opt. Soc. $A m ., 1957,47,120$ (SA17). Hunter (W. R.), Optica Acta, 1962, 9, 255.

[12] Madden (R. P.), Canfield (L. R.) and Hass (G.), J. Opt. Soc. Am., 1963, 53, 620.

[13] LaVilla (R.) and Mendlowitz (H.), Phys. Rev. Ltrs, $1962,9,149$

[14] LaVilla (R.), Private communication.

[15] Tomboulian (D. H.) and Bedo (D. E.), Rev. Sc. Inst., 1955, 26, 747.
[16] Astoin (N.) and Vodar (B.), J. Physique Rad., 1953, $14,424$.

[17] Tomboulian (D. H.) and Pell (E. M.), Phys. Rev., $1951,83,1196$.

[18] Hass (G.) and Waylonis (J. E.), J. Opt. Soc. Am., $1961,51,719$.

[19] Walker (W. C.), Rustigi (O. P.) and Weissler (G. L.), J. Opt. Soc. Am., 1959, 49, 1471.

[20] Frohlich (H.) and Pelzer (H.), Proc. Phys. Soc., $1960,75,664$.

[21] Mendlowitz (H.), J. Opt. Soc. Am., 1960, 50, 739.

[22] Robins (J. L.), Proc. Phys. Soc., 1962, 79, 119.

[23] Wilkinson (P. G.), Private communication.

[24] Marton (L.), Leder (L. B.) and Mendlowitz (H.), Ads. in Electronics and Electron Phys., 1955, 7, 183.

[25] Kroger (H.) and Tomboulian (D. H.), Phys. Rev., $1963,130,152$

[26] Sasaki (T.) and Ishiguro (K.), Phys. Rev., 1962, 127, 1091.

[27] Madden (R. P.), The Physics of Thin Films, 1963, 1, 123.

[28] Handbook of Chemistry and Physics, Chemical Rubber Publ. Co., Cleveland, Ohio, 1959.

[29] Sandstrom (A. E.), Hanb. Physik, 1957, 30, 78, Berlin : Springer-Verlag, Ed by S. Flugge. 